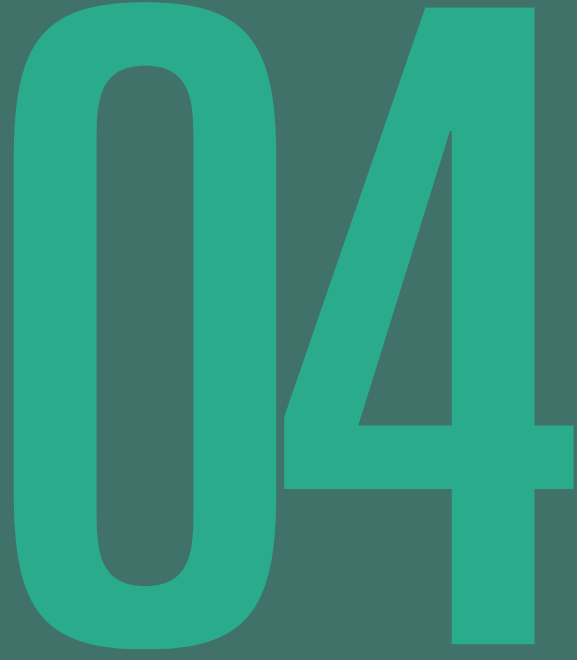

\title{
PROPUESTA DE \\ INSTRUMENTO PARA MEDIR IDENTIDAD A PARTIR DEL MOBILIARIO URBANO
}

\section{Proposal for an instrument to measure identity from urban furniture}

Beatriz Paredes de la Rosa

Estudiante de la Maestría en Ciencias con Orientación en Gestión e Innovación del Diseño en la Facultad de Arquitectura de la Universidad Autónoma de Nuevo León. Arquitecta por la Universidad Tecnológica de la Habana (UTH) Cujae, Cuba.

Dra. Liliana Beatríz Sosa Compeán https://orcid.org/0000-0001-8811-3218

liliana.sosacm@uanl.edu.mx

Doctor en Creación y Teorías de la Cultura (UDLAP), Profesor de tiempo completo en la Universidad Autónoma de Ciudad Juárez (UACJ), Coordinador de la Maestría en Estudios y Procesos Creativos en Arte

y Diseño y Líder del Cuerpo Académico, Diseño, Usuario y Entorno 
Palabras clave: mobiliario urbano, identidad urbana, sistema complejo, lectura urbana, percepción, instrumento de medición.

Keyword: urban furniture, urban identity, complex system, urban reading perception, measuring instrument.

Resumen:

La identidad urbana es un tema recurrente dentro del diseño urbano, ya que define la vinculación de un grupo bumano con su entorno y el nivel de arraigo presente en un barrio o ciudad. Un espacio público donde las inserciones (como el mobiliario) fomenten ese sentido de pertenencia sería una máxima en el diseño urbano. Pero ¿cómo se debería diseñar mobiliario para este fin?, ¿qué factores intervienen en el proceso de diseño del mobiliario urbano que genere identidad? y ¿cuáles deberían ser las estrategias a seguir?, ¿cómo se podría saber si un elemento urbano fomenta identidad? Por estas y otras interrogantes se consideró necesario crear un instrumento que ayude a medir el grado de pertenencia $y$, por ende, a identificar interacciones significativas en las relaciones sujeto-objeto, señalando que lo antes mencionado se estudia bajo un enfoque holístico donde el pensamiento sistémico ayude a minimizar las visiones reduccionistas.
Abstract:

Urban identity is a recurring theme within urban design as it defines the connection of a buman group with its environment and the level of roots present in a neighborhood or city. A public space where insertions (such as furniture) foster that sense of belonging would be a maxim in urban design. But how should furniture be designed for this purpose? What factors are involved in the process? What should be the strategies to follow? How could you know if an urban element fosters identity? Due to these and other questions, it was believed necessary to create an instrument that helps to measure the degree of belonging and therefore to identify significant interactions in subject-object relationships. All of the aforementioned is studied under a bolistic approach where systems thinking helps to minimize reductionist views. 
Beatriz Paredes de la Rosa

Liliana Beatríz Sosa Compeán

\section{INTRODUCCIÓN}

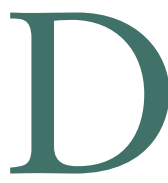

esde las comunidades primitivas se identifica el predominio de las características de un grupo social sobre las particulares de cada individuo de dicho grupo. Según Oguri, (s.f.) con el desarrollo y crecimiento de las sociedades, la división social del trabajo, así como la globalización en la cual nos encontramos, fue complejizando cada vez más la construcción de identidades.

Las relaciones de la sociedad en un espacio público generadas por elementos urbanos definen a grandes rasgos el fenómeno de la identidad urbana. El mobiliario como uno de estos elementos constituye parte esencial del paisaje urbano. En estos espacios físicos se evidencian las sensaciones de un lugar identitario donde la cultura, el espacio construible y los habitantes dialogan, generando tradiciones (Oguri, s.f.).

Según Morgan Ball, (2006) el ciudadano debe ser considerado un elemento importante del paisaje urbano y por esta razón las intervenciones en los espacios de confluencia social deben ser analizadas cuidadosamente para 
contribuir a las relaciones y a su permanencia como usuario. Es por ello que se considera necesario contar con herramientas que posibiliten, de alguna forma, medir la identidad en los espacios públicos. Con ello se pretende identificar los elementos que contribuyen a la generación de un sentido de pertenencia hacia estos espacios para poder entonces gestionar las inserciones en el mobiliario urbano que fomenten identidad en barrios y ciudades.

Surge así, como objetivo principal de este artículo, proponer una postura para determinar cómo medir el fenómeno de la identidad urbana a partir del mobiliario urbano de acuerdo con un enfoque holístico en el que se integren diversas teorías estudiadas. En una primera etapa del estudio se integran las visiones del pensamiento sistémico y la complejidad para entender a la ciudad, así como a la lectura de esta y la identidad urbana. Se estudia entonces a la ciudad como sistema complejo para evitar el enfoque reduccionista e interrelacionar los actores y factores que hacen que exista identidad en ella. De igual manera, se analiza la percepción del mobiliario urbano para analizar qué factores son relevantes en el diseño de estos elementos. Y, por último, da cabida a la lectura urbana como el proceso de significación y comprensión de los factores que van a determinar la identidad urbana. Finalmente se presenta una propuesta de instrumento para medir identidad urbana teniendo en cuenta los postulados relevantes analizados en la primera etapa y brindando algunas consideraciones de aplicación.

\section{LA CIUDAD, LA COMPLEJIDAD, LA IDENTIDAD Y LA LECTU- RA URBANA}

En la actualidad los crecimientos desmedidos de las ciudades traen, entre muchos otros problemas, la pérdida de la identidad urbana. En este constante desarrollo urbano se descuida, en muchas ocasiones, la planificación a escalas; definida no solo por las escalas de diseño, sino por las escalas espaciales que delimitan barrios, colonias, ciudades y estados. Este concepto de identidad permite establecer relaciones físicas entre elementos de diseño y relaciones sociales que sobrepasan los límites del marco construido generando las dinámicas sociales que le dan vida a la ciudad. 
El enfoque con que se trata el tema del diseño de las partes componentes de una ciudad en la mayoría de los casos es reduccionista. En estos no se enfocan las soluciones ni el análisis de los elementos entrelazados con el resto del conjunto urbano, sino que se suele separar del resto del entorno. Gaja I Diaz, (2011) afirma: "El urbanismo de la modernidad maltrató el espacio público, condenándolo a su extinción. El espacio público fue reducido a lo que quedaba entre los edificios: tierra de nadie, espacio marginal, indiferenciado, desestructurado" (p.166).

El autor asevera, dentro de su catálogo de agresiones al espacio público, que éste ha sido privado de su característica principal que es ser un espacio social. Éste ha sido convertido en un armazón o soporte funcional, pero con más orientación en la actualidad hacia el soporte de una movilidad motorizada. Las funciones relativas a la sociedad, dígase espacio de encuentro y reunión, lugar de recreación y esparcimiento, soporte de relaciones sociales y culturales, espacio de sociabilización e integración, entre otros se han visto limitados en la actualidad (Gaja I Diaz, 2011).

En consonancia con la importancia del estudio del tema Muñoz Villalobos y Catalán Neumann (2015) plantean:

Genera preocupación una ciudad con un grupo humano desvinculado con su entorno inmediato y carente de arraigo con su localidad y contribuye a transformar la ciudad en un sitio opaco, con una historia silenciada que no da cuenta de su relevancia real e histórica. (p.2)

Un espacio público diseñado de forma tal que fomente la identidad urbana va a potenciar el sentido de pertenencia en la sociedad. La cohesión social, el respeto hacia el medio físico construido y el cuidado medioambiental del espacio público y, por ende, la ciudad serían algunos de los muchos beneficios generados por este sentido de pertenencia.

Por lo antes mencionado es que la medición deberá considerar aspectos como las escalas espaciales y diferenciaciones de espacios, sin descuidar las relaciones entre éstos. Además, se debe prestar vital atención a la vocación social de los espacios públicos, es decir, la interacción del grupo humano estudiado con el entorno. 
De esta manera, se consideró pertinente el estudio de la ciudad como sistema complejo, definido como el conjunto de elementos en relación capaces de adaptarse, modificarse y reestructurarse bajo diferentes estímulos y circunstancias. Un sistema complejo adaptativo (SCA) es una red dinámica de muchos agentes interactuantes. El resultado total del sistema tiene un comportamiento coherente que proviene de un enorme número de decisiones hechas en algún momento por muchos agentes individuales. Lo que distingue a un SCA de un simple sistema multiagente es el enfoque en propiedades y características de alto nivel como la autosimilitud (el sistema es similar a partes de él mismo, tiene procesos similares en distintas escalas o niveles), la complejidad, la emergencia y la autoorganización. (Holland, 1992).

Estos sistemas son de particular interés porque, dependiendo de su naturaleza, pueden representar células, especies, individuos, cerebros, empresas, naciones, colonias de hormigas o ciudades. Aunque el control de un SCA tiende a ser altamente disperso y descentralizado, entender a los sistemas que se estudian con este enfoque y entender sus mecanismos, propiedades y características resulta útil cuando se desean lograr objetivos (como identidad y sentido de pertenencia) a partir de intervenirlos (con mobiliario urbano, por ejemplo, en nuestro caso). Para entender los mecanismos particulares de un sistema social como en el que en nuestro caso se desea intervenir, los instrumentos deberán contemplar las características y condiciones de los objetos y el entorno; pero, sobre todo, las interacciones que suceden en los espacios. La relación de las partes de un sistema genera comportamientos cooperativos y además se presentan en escalas de jerarquía que repercuten directamente en la organización y buen funcionamiento del conjunto. Se estudió a la ciudad como sistema complejo para no divorciar el objeto de diseño del contexto y poder interrelacionar los actores y factores que hacen que exista identidad en la ciudad, mediante el estudio de las dinámicas sociales que se generan a partir del uso del mobiliario y los espacios.

El uso del espacio urbano y los objetos que lo componen por la sociedad genera información y apoya su transmisión. Ya sea el sentarse en una banca, el esperar un transporte público en una parada, el leer un cartel informativo, el 
beber agua de un bebedero, el recorrer un camino alumbrado en la noche, el usar el cesto de basura o jugar en un aparato infantil genera comportamientos o patrones en la sociedad. Es el significado tangible o perceptual que generen estos elementos lo que determina la relación en torno a ellos y es precisamente mediante la interacción objeto-sujeto. Según Latour (2005) los objetos actúan como medidores activos capaces de generar transformaciones en la sociedad. Para esto en su teoría del actor-red, plantea que un fenómeno social no puede ser estudiado sin considerar las interacciones de personas y cosas.

Estos componentes o actores en interacción generan datos e información que pueden verse reflejados en palabras, sentimientos, emociones, comportamientos, valores, entre otros. Todos estos datos nutren a la ciudad y la ayudan a auto regenerarse y producirse. Por consiguiente, el instrumento de medición deberá estar enfocado en gran medida a obtener este tipo de datos mediante escala de emociones, usos, así como medición de interacciones entre el usuario, el espacio y los elementos componentes.

Complementando estos temas se analiza además cómo el usuario del espacio público ve y siente tanto el lugar como sus elementos componentes. Se consideró para estudiar también a la percepción del mobiliario urbano con el propósito de analizar qué factores son relevantes en el diseño de estos elementos. Con estos conceptos se puede entonces llegar a comprender cómo es percibido el mobiliario urbano por la sociedad.

La percepción es la forma que tiene una persona de captar a través de los sentidos, las sensaciones y estímulos del entorno. Mediante el procesamiento de éstos, un usuario o conjunto de ellos, puede conformar una impresión de la realidad influenciada además por experiencias anteriores o perspectivas particulares. La percepción del mobiliario urbano en el espacio público sería entonces la impresión que generan estos elementos de diseño desde un medio físico construido y hacia la sociedad. Es esta la que sería necesario detectar en este tipo de instrumento de medición ya que lo que perciba el usuario de 
un medio físico construido va a incidir directamente en su apropiación por éste y particularmente por los elementos que lo conformen, en este caso, el mobiliario urbano.

Por otro lado, Oguri (s.f.) identifica los tres aspectos fundamentales en los que radica la importancia del mobiliario urbano: lo contextual, lo funcional y la cantidad y ubicación. Además, identifica los factores que se deben tener en cuenta para su diseño, donde los cuatro principales son: funcionalidad, resistencia, producción, estética y ergonomía. Estas características del mobiliario urbano deben ser medidas en el instrumento de manera tal que se contraste con la percepción que tenga el usuario de estos elementos urbanos. Es decir, tratar de saber si el ciudadano identifica, al igual que los urbanistas, diseñadores y arquitectos, estas características y cuáles son las que le hacen sentir mayor afecto hacia el espacio y el mobiliario.

Para la medición de identidad a partir del mobiliario urbano también se consideró a la lectura urbana como el proceso de significación y comprensión. La identidad es una característica relativa a elementos mediante la cual se pueden crear asociaciones dentro de un entorno, a través de la distinción, o lo que es lo mismo, la identificación de características similares y diferentes. En el caso de las ciudades, esta transmisión de información hacia los ciudadanos se da mediante sus elementos componentes y las características que le confiere la llamada identidad urbana. Este fenómeno se define como la lectura que genera un espacio, entorno o ciudad hacia los seres humanos.

En su libro, La imagen de la ciudad, Lynch (1998) define tres términos para este fin, la identidad, la estructura como relación espacial del objeto con el observador y demás objetos y, por último, el significado de estos, ya sea práctico o emotivo. Define que el reconocimiento de una identidad separable, es decir su distinción respecto a otras, sería el primer término para una imagen eficaz. 
Ahondando más sobre el concepto y las teorías que sustentan el tema, se debe mencionar a los autores Sergio Tamayo y Kathrin Wildner (2005) y su libro Identidades Urbanas. En su texto, los autores refieren que para definir identidad se basan en cuatro elementos: reconocimiento, pertenencia, permanencia y vinculación.

Por lo tanto, el instrumento debe estar orientado a identificar estos elementos en el comportamiento del usuario en el espacio. Es decir, saber si estos reconocen al espacio público y al mobiliario como suyo, si sienten pertenencia por éstos y, de ser así, de qué forma o mediante qué factores se da esta vinculación. Lo anterior pudiera medirse mediante el conocimiento del significado que tengan estos elementos para el usuario y las emociones que experimenta con el uso de éstos.

Mercedes Mercado Cisneros (2012) en su tesis doctoral, La identidad de las ciudades a través de los objetos urbanos, identifica en una síntesis de estudio de teorías de la identidad tres ejes fundamentales relativos a ésta: la inclusión-exclusión, la coercitiva-persuasiva y la objetividad-subjetividad. Estos aspectos pudieran tenerse en cuenta a la hora de analizar los espacios que serían objeto de estudio o aplicación del instrumento.

Con las teorías analizadas se estructuraron entonces algunas pautas a tenerse en cuenta en la conformación del instrumento para medir identidad a partir del mobiliario urbano. A continuación, se describirá a grandes rasgos cómo podría ser la estructura y confección de éste, se expondrá un ejemplo de cómo podría quedar para su aplicación, además de algunas recomendaciones que podrían tenerse en cuenta.

\section{MATERIALES Y MÉTODOS}

El procedimiento que se siguió para el desarrollo de este trabajo se estructura a través de seis pasos, los cuales fueron los siguientes: 
1. Establecimiento del marco teórico referencial: Se realizó una investigación documental para abordar desde el enfoque sistémico a la identidad en las ciudades y cómo se establecen las relaciones socio-espaciales en el espacio público y con el mobiliario urbano en particular. De esta primera etapa derivaron pautas a seguir y tener en cuenta a la hora de confeccionar una primera propuesta de instrumento de medición.

2. Definición de variables para el instrumento: Con base en el marco teórico referencial se determinaron las secciones que deben integrar el instrumento atendiendo a las escalas del sistema ciudad y a las interacciones relevantes entre sujetos y objetos. De esta forma se puntualiza en la necesidad de estudiar a la muestra escogida para la aplicación del instrumento, medir el grado de interacción del usuario con el espacio público, estudiar la interacción social que pueda darse en este, así como la visión del espacio público y el mobiliario urbano por el ciudadano.

3. Propuesta de indicadores a medir: Mediante la operacionalización de variables y las pautas fijadas con anterioridad se definieron los indicadores a medir en el instrumento. Estos fueron determinados, además, por secciones para no perder el enfoque dentro de cada una de ellas. Con los indicadores se pretende determinar el grado de integración social y sentido de pertenencia del grupo humano estudiado con el espacio público mediante el estudio de las frecuencias de usos, el tiempo de permanencia, el grado de participación en las actividades, la escala básica de emociones del usuario en interacción con el mobiliario y el sitio, entre otros.

4. Propuesta de ítems: Mediante referentes preexistentes y lluvia de ideas se determinaron series de preguntas para cada sección que pudieran reflejar los indicadores que se buscan medir. Estas en su mayoría son de respuestas de tipo escala de Likert facilitando así la aplicación y procesamiento. Por cada sección, además de este tipo de preguntas, se determinaron otras del tipo respuesta abierta que ayudan a tener una visión más integral de lo que siente el usuario en el espacio.

5. Validación de Instrumento de medición: Se realizó un pilotaje que consistió en la aplicación de la propuesta preliminar de instrumento de medición a una muestra de diez usuarios del espacio público de diferentes edades y 
sexos. Las respuestas obtenidas fueron procesadas mediante el programa estadístico SPSS para obtener el grado de fiabilidad mediante el estadístico de Alfa de Cronbach. De esta manera se pudieron identificar los ajustes necesarios para mejorar la fiabilidad.

6. Establecimiento del instrumento de medición: Luego de ajustar las preguntas y eliminar las que interferían con la validez del instrumento se llegó a la propuesta final, la cual se describirá a continuación.

\section{INSTRUMENTO PARA MEDIR IDENTIDAD A PARTIR DEL MOBILIARIO URBANO}

Lo antes visto nos llevó a la propuesta de un instrumento de medición en forma de encuesta. Esta se aplicaría a usuarios del espacio público en espacios físicos delimitados y definidos. En caso de ser más de un espacio, éstos deberán tener características diferentes atendiendo a extensión territorial, escala, ubicación, morfología, centralidad urbana, funciones y actividades recreativas presentes. De esta forma se podrán contrastar valores y datos obtenidos para correlacionarlos.

En la estructura de la encuesta se deben tener en cuenta las consideraciones mencionadas a lo largo de la investigación. Conviene entonces dividirla en secciones que posibiliten la comprensión del encuestado de la información requerida. Algunas de estas pudieran estar definidas por tipos de interacción, por ejemplo, sujeto-espacio, sujeto-objeto, sujeto-sujeto en el espacio. Se recomienda además que las preguntas que predominen sean de selección múltiple para así facilitar la aplicación y acortar los tiempos de respuestas. Esto no debe interferir con la libre expresión del usuario sobre su percepción, por lo que, para la medición de ésta, se deben dejar algunas preguntas abiertas puntuales.

Para aplicar una encuesta con preguntas de selección múltiple se deben establecer rangos y escalas. Así, se determinarán rangos continuos, de intervalos cortos para facilitar la medición y establecer medias o tendencias. Por ejemplo, para medir tiempos de permanencia, traslado y uso se pudieran utilizar los intervalos en horas de dos en dos. En el caso de preguntas que responden a 
escalas se deberán utilizar continuas en su organización facilitando la comprensión del lector, como, por ejemplo, la escala básica de emociones o la de medir calidad (excelente, bueno, regular o malo).

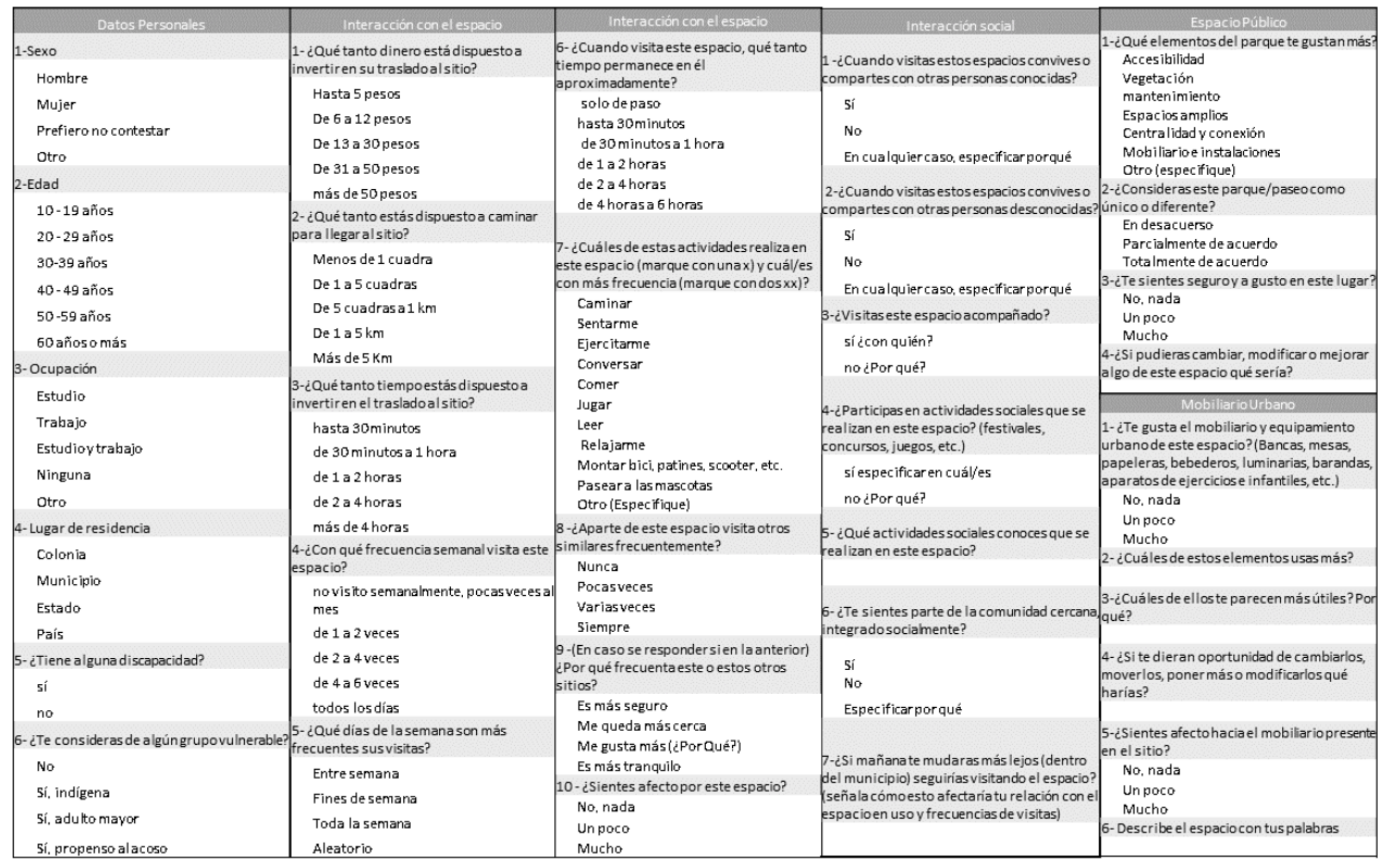

Ilustración 1 Propuesta de instrumento de medición. Elaboración propia.

Teniendo en cuenta la información brindada hasta ahora, se propone un ejemplo de encuesta que considere todos estos aspectos y facilite la visualización de la medición.

La encuesta propuesta está dividida en secciones de acuerdo con los temas que se vayan a estudiar y medir (ver Ilustración 1). Se pretende que sean lo más dinámicas posibles, predominando las preguntas de escalas y selección múltiple como se mencionó anteriormente. Se compone de 5 secciones. La primera está destinada a recopilar información del encuestado para así crear perfiles; la segunda va dirigida a medir el grado de interacción del usuario con el espacio público; la tercera va destinada a estudiar la interacción social que pueda darse en éste; la quinta se dedica especialmente a la visión del espacio público por el ciudadano y la sexta específicamente al mobiliario urbano. 


\section{DISCUSIÓN Y RESULTADOS}

A partir de la propuesta preliminar del instrumento de medición a partir del mobiliario urbano, desarrollada a partir del estudio teórico de las variables mencionadas, se procedió a su validación con la realización de una prueba piloto. Para la validación del instrumento de medición se dividió en secciones el análisis de fiabilidad mediante el estadístico Alfa de Cronbach. Es aconsejable realizarlo de esta manera debido a que el estadístico interpreta las preguntas que se analizan como un puntaje único sin discriminar si estas responden a temas diferentes, lo que se conoce como unidimensionalidad. En el instrumento se utilizaron siete ítems para analizar la interacción social, diez ítems para analizar la interacción con el espacio y cuatro para analizar el espacio público y al mobiliario urbano. Por ello fue conveniente estudiarlos por separado obteniendo así adecuados índices de fiabilidad. (ilustraciones 2, 3 y 4)

\begin{tabular}{|c|c|}
\hline \multicolumn{2}{|c|}{ Estadísticos de fiabilidad } \\
\hline $\begin{array}{c}\text { Alfa de } \\
\text { Cronbach }\end{array}$ & $\begin{array}{c}\mathrm{N} \text { de } \\
\text { elementos }\end{array}$ \\
\hline .750 & 7 \\
\hline
\end{tabular}

Ilustración 2 Estadístico de fiabilidad 1. Para los ítems de interacción social. Elaboración Propia

\begin{tabular}{|c|c|}
\hline \multicolumn{2}{|c|}{ Estadísticos de fiabilidad } \\
\hline $\begin{array}{c}\text { Alfa de } \\
\text { Cronbach }\end{array}$ & $\begin{array}{c}\mathrm{N} \text { de } \\
\text { elementos }\end{array}$ \\
\hline .722 & 10 \\
\hline
\end{tabular}

Ilustración 3 Estadístico de fiabilidad 2. Para los ítems de interacción con el espacio. Elaboración Propia

\begin{tabular}{|c|c|}
\hline \multicolumn{2}{|c|}{ Estadísticos de fiabilidad } \\
\hline $\begin{array}{c}\text { Alfa de } \\
\text { Cronbach }\end{array}$ & $\begin{array}{c}\text { N de } \\
\text { elementos }\end{array}$ \\
\hline 810 & 4 \\
\hline
\end{tabular}

Ilustración 4 Estadístico de fiabilidad 3. Para los ítems del análisis del espacio público y mobiliario urbano. Elaboración Propia

Algunos de los resultados obtenidos con el pilotaje responden además a la repercusión de las preguntas en la confiabilidad del instrumento. Se determinaron que las que más fiabilidad aportan son las referentes a la convivencia e integración a la población circundante y visitante; a los tiempos de traslado, permanencia y uso de los elementos urbanos; al afecto que tienen los usuarios hacia el mobiliario y el espacio público, así como a las actividades que realizan.

Lo antes expuesto tal vez se deba principalmente a la facilidad con que los encuestados responden a preguntas con las que se encuentran más familiarizados, así como el lenguaje que se utiliza al ser menos científico y más atemperado con el usuario. De igual manera se determinaron las preguntas que pudieran disminuir la fiabilidad del instrumento tales como las referentes a sentirse seguro y protegido en el espacio público, así como pertenecer a un grupo vulnerable; las que indicaban cuánto dinero se estaba dispuesto a 
invertir en el traslado al sitio y las de preferencia de otros espacios públicos urbanos diferentes al que se presentaba el usuario cuando se realizó el pilotaje. Lo anterior podría deberse a que en éstas preguntas se tocan temas sensibles, que no todos los encuestados están dispuestos a responder o simplemente no se sienten cómodos hablando de cuestiones monetarias ni de vulnerabilidad, apoyado además por la situación actual de la seguridad en la ciudad en general. Se recomienda tener en cuenta estos resultados en la conformación de nuevos instrumentos de medición siempre considerando qué se desea medir ya que eliminar alguna pregunta de las antes mencionadas pudiera afectar el logro de los objetivos de la investigación.

Es válido mencionar que se debe definir la etapa de aplicación del instrumento atendiendo al tipo de proyecto a llevar a cabo ya sea de investigación o proyectos de construcción. Además, se debe considerar el alcance de este para su uso oportuno. Con una aplicación en el tiempo correcto se pudieran advertir problemas futuros o determinar estrategias de intervención que efectivamente contribuyan a fomentar la identidad en los espacios urbanos objetos de estudio.

\section{CONCLUSIONES}

Con el trabajo, se pudieron determinar algunos factores que intervienen en el proceso de diseño de este: a) la consideración de la percepción de los usuarios b) las escalas espaciales c) la interrelación de los objetos en el espacio d) la interacción del grupo social que habita el espacio y el entorno e) la consideración de las emociones de los usuarios y f) su significación para los usuarios $\mathrm{y}$, con esto, conocer si contribuyen o no al fomento de la identidad urbana.

Se concluye que para medir el grado de identidad que generan los objetos urbanos pueden considerarse: 1) el comportamiento del usuario en el espacio 2) el tipo de uso que se le da al espacio 3) el tiempo de permanencia 4) el reconocimiento del espacio como suyo para determinar sentido de pertenencia y 5) las escalas de emociones que genera la interacción con el mobiliario.

Con un instrumento como éste se hace necesario hacer una correlación de los datos obtenidos en la aplicación con un análisis detallado de los espacios en los cuales se aplicaría. Es recomendable complementar con mapeos de los 
sitios y delimitaciones de escalas dentro de estos, sobre todo, en espacios de grandes dimensiones donde convergen otros de menores dimensiones. Además, se debe hacer un levantamiento del mobiliario existente en estos y de sus características formales y funcionales para así tener una comprensión más cercana a la realidad de las respuestas obtenidas con la encuesta.

Otra forma de contrastar la información podría ser la investigación en línea de los lugares estudiados y el análisis de las opiniones que de estos se dan mediante la netnografía. Debe considerarse que este método se ajustaría mejor a espacios de escalas más grandes, más visitados o de más impacto en los barrios y ciudades. Al generarse con ellos mayor tráfico digital de información en redes pudieran obtenerse respuestas más diversificadas o mayor número de estas.

Con todo lo anterior se puede decir que es factible medir el grado de pertenencia que los ciudadanos tienen hacia un espacio público e identificar las interacciones significativas en las relaciones que presentan con el medio. Todo esto para brindar herramientas a los investigadores, diseñadores, arquitectos y urbanistas que se enfrenten tanto a la planificación urbana como al diseño de mobiliario urbano en el fomento de la identidad. 


\section{REFERENCIAS}

Gaja i Diaz, F. (2011). El espacio público una especie amenazada. Catálogo de agresiones, decálogo para su recuperación. Ciudades (im)propias: la tensión entre lo global y lo local: (165-178). Valencia: Centro de Investigación Arte y Entorno; Universitat Politècnica de València.

Holland, J. H. (1992). Complex adaptive systems. Daedalus, 121(1), 17-30. Recuperado de http://www.jstor.org/stable/20025416

Latour, B. (2005). Reensamblar lo social: una introducción a la teoría del actor-red. Buenos Aires: Ediciones Manantial.

Lynch, K. (1998). La imagen de la ciudad. Barcelona: Editorial Gustavo Gili.

Mercado Cisneros, M. (2012). La identidad de las ciudades a través de los objetos urbanos. (Tesis de doctorado). Universidad Autónoma de Nuevo León, Monterrey. Recuperado de http://eprints.uanl.mx/id/eprint/5945

Morgan Ball, D. (2006). Los usuarios del espacio público como protagonistas en el paisaje urbano. Revista de Arquitectura, 8(1), 34-41. Recuperado de http:// editorial.ucatolica.edu.co/ojsucatolica/revistas_ucatolica/index.php/RevArq/ article/view/807/837

Muñoz Villalobos, D., y Catalán Neumann, R. (2015). Percepción urbana: Imagen de la ciudad de Talca según población adolescente. NADIR: Revista electrónica geografía Austral, 7(2). Recuperado de http://revistanadir.yolasite.com/año7-nº-2-agosto---diciembre-2015.php

Oguri, L. (s.f.). La identidad cultural y su reflejo en el mobiliario urbano: estudio de caso Ixtapamde la sal. Universidad Autónoma del Estado de México. Recuperado de https://www.academia.edu/4415494/Reflejo_de_la_identidad_cultural_en_el_mobiliario_urbano

Tamayo, S. y Wildner, K. (2005). Identidades Urbanas. México: Universidad Autónoma Metropolitana. 